\title{
Aporte económico de la madera de cedro (Cedrela odorata L.) como árbol de sombra en cafetales de Pérez Zeledón, Costa Rica
}

\section{Economic contribution of cedar wood (Cedrela odorata L.) as a shade tree in coffee plantations in Pérez Zeledón, Costa Rica}

\author{
Sergio Calvé Jarque ${ }^{1}$ (D) $\bullet$ Olman Murillo² (D) • Donald Córdoba ${ }^{3}$ (D) • Luis Salazar³
}

Recibido: 29/10/2019 Aceptado: 5/05/2020Ｐublicado: 28/07/2020

\begin{abstract}
The study determined the economical contribution of cedar wood (Cedrela odorata L.) in agroforestry systems with coffee in Costa Rica. 26-coffee farms associated to CoopeAgri R.L. in Pérez Zeledón county. Cedar has been planted as a shade tree in coffee production areas in this county, with an average density of 214 trees/ha, which corresponds to a $7 \times 7 \mathrm{~m}$ spacing, but it varies from 70 to 700 trees. Cedar in coffee plantations registers a growth rate of $2.5 \mathrm{~cm} /$ year in diameter, which allows it to reach $40 \mathrm{~cm}$ in dbh at 16 years of age. Planting cedar in coffee areas produce economical incomes from wood sales of approximately $\$ 5000 \mathrm{ha}^{-1}$ and a total commercial volume of $100 \mathrm{~m}^{3} \mathrm{ha}^{-1}$ in 16 years. From this amount $60 \%$ has commercial value for sawmill industry in its $6 \mathrm{~m}$ merchantable stem. Meanwhile, fuelwood represents $35 \%$ of total volume, but only $8 \%$ of the total tree value. Good silvicultural management techniques, like crown prunning and Hypsipyla grandella attack prevention, with an initial density of 277 trees ( $6 \times 6 \mathrm{~m}$ that will be thinned to $12 \times 6 \mathrm{~m}$ at age 8 ) may increase the wood value up to $\$ 7000 \mathrm{ha}^{-1}$. Cedar trees planted in agroforestry systems with coffee, is an excellent option that enhances higher incomes for producers.
\end{abstract}

Key words: Forest economics, volume, valuation, agroforestry.

1. Universidad Politécnica de Valencia. ETSIAMN. Valencia, España; calvejarquesergio@gmail.com

2. Instituto Tecnológico de Costa Rica. Escuela de Ingeniería Forestal, Cartago, Costa Rica; olmuga@yahoo.es

3. Corporación Coopeagri R.L., Pérez Zeledón, Costa Rica; Isalazar@coopeagri.co.cr, dcordoba@coopeagri.co.cr. 


\section{Resumen}

La investigación estimó el aporte económico de la madera de Cedro (Cedrela odorata L.) por medio de árboles de sombra en sistemas de producción de café. Se censaron lotes productivos en 26 cafetales de productores asociados a la Corporación Coopeagri R.L en el cantón de Pérez Zeledón. El cultivo de cedro en cafetales de la región se maneja a una densidad promedio de 214 individuos ha-1, que corresponde a un espaciamiento de $7 \mathrm{~m} \times 7 \mathrm{~m}$, no obstante, se registró una significativa variación desde 70 hasta 700 individuos. El cedro en cafetales tiene una tasa de crecimiento de $2,5 \mathrm{~cm}$ año-1, que le permite alcanzar los $40 \mathrm{~cm}$ en el DAP a los 16 años de edad. El cultivo de cedro en los cafetales produce ingresos económicos por la madera, de aproximadamente $\$ 5000 \mathrm{ha}^{-1} \mathrm{y}$ un volumen comercial de $100 \mathrm{~m}^{3}$ ha $^{-1}$ en 16 años, del cual, el $60 \%$ tiene valor comercial para aserrío en 6 a $7 \mathrm{~m}$ de fuste. La leña representa un $35 \%$ del volumen total, pero tan solo un $8 \%$ del valor comercial del árbol. Con buenas prácticas silviculturales de manejo de copa y de reducción del ataque de Hypsipyla grandella, con una densidad inicial de 267 árboles ( $5 \times 7,5 \mathrm{~m}$ que se reduce a 160 al año 8$)$, el valor de su madera podría alcanzar los $\$ 7000$ ha $^{-1}$. El cedro en sistemas agroforestales asociado con café es una excelente opción de diversificación productiva para sus productores.

Palabras clave: Economía forestal, volumen, valuación, agroforestería.

\section{Introducción}

El café en Costa Rica es la actividad económica con mayor trascendencia a lo largo de la historia, con influencia en el desarrollo del modelo socioeconómico del país, hasta casi finales del siglo XX [1]. El café siempre ha sido base fundamental y motor para el desarrollo económico, social y cultural de Costa Rica. Reconocido como "El Grano del Oro" y considerado como uno de los mejores del mundo por su calidad. Costa Rica cuenta con un estimado de 84133 ha cultivadas de café [2] de las que dependen más de 50 mil familias productoras y 290 beneficios. El sector cafetalero representa aproximadamente el $8 \%$ de la fuerza laboral costarricense. En la cosecha 2015-2016 aportó un 11,5 \% del Producto Interno Bruto Agrícola (PIB), correspondiente a 8,9 \% de la producción total de café en Centroamérica [3]. El país exporta un $82 \%$ del total de café producido, con lo que genera un alto impacto en la economía nacional [4]. En el cantón de Pérez Zeledón, Pacífico central del país, la actividad cafetera es fundamental donde se registran más de 4500 fincas dedicadas a esta actividad, con un promedio de menos de 3 ha por productor [2]. En su mayoría están incorporados a la Corporación Coopeagri R.L. (Cooperativa Agrícola e Industrial de servicios múltiples el General R.L.), donde se promueve una caficultura sustentable basada en sistemas agroforestales, en asocio con cedro, cítricos, musáceas, cacao y otras plantas [5]. El asocio del cultivo del café con árboles de cedro y laurel, ha sido una práctica convencional de amplia utilización no solo en Costa Rica [6], [7], [8], sino en países como Colombia, México, Guatemala y Honduras [9], [1], [10], [11], [12].

El cedro amargo (Cedrela odorata) es una madera muy utilizada en Costa Rica para la elaboración de muebles, decoración de interiores, lanchas, puertas, instrumentos musicales, armarios y cajas para el almacenamiento [4]. De acuerdo con estadísticas de la Oficina Nacional Forestal [13], la madera del cedro amargo sigue posicionada entre las 10 especies de mejor precio en el mercado nacional. Algunos estudios han reportado al cedro con una tasa relativamente alta de crecimiento en plantación, donde se menciona que puede llegar a alcanzar cerca de los 30 $\mathrm{cm}$ en 10 años de edad en condiciones óptimas [1], [14]. La caficultura ofrece por tanto, un espacio prometedor para la producción sostenible de madera de alta calidad en toda las regiones cafetaleras del país.

La modalidad de café con sombra en Costa Rica hace posible que surjan otros cultivos que puedan asociarse y permitan diversificar los ingresos del caficultor convencional. En los últimos años, los fuertes cambios en el precio internacional del grano tienen al sector en continuas crisis, que han provocado el colapso de algunas organizaciones y de muchos productores. Junto con los vaivenes del precio, los problemas fitosanitarios como la roya y el ojo de gallo, reducen significativamente la productividad, que también inciden en la disminución de los ingresos económicos de los productores [15]. Como medida de mitigación, se ha propuesto mejorar el sistema de café con sombra (SAF), de modo que se logre un mayor ingreso e impacto económico-social de la actividad.

Como efecto directo se espera que los SAF con café incidan positivamente en una mejora de los servicios ambientales, por ejemplo aumento en la biodiversidad, tasa de fijación de carbono y se logre mitigar sus emisiones [4]. La producción de café en Costa Rica está íntimamente ligada a la identidad del país y proporciona empleo hasta a 150000 personas durante la cosecha. Sin embargo, puede generar hasta un 1,56\% de las emisiones nacionales de gases de efecto invernadero (GEI). La huella de carbono de la actividad se ha determinado como negativa, debido al componente de beneficiado del café y al uso excesivo de fertilizantes nitrogenados. Por tal razón, el uso correcto de la sombra puede ayudar a mitigar la utilización de fertilizantes en el cafetal [16], 
en concordancia con las políticas nacionales del NAMA café (Acciones Nacionales de Mitigación Adecuadas). El cultivo de maderables en el cafetal permitirá también aumentar la producción de materia prima, que podría ser utilizada en la generación de energía renovable. Con esto se podría lograr una disminución importante en los costos de beneficiado del café.

EI SAF café ha sido abordado tradicionalmente con mayor énfasis en sus servicios ambientales, como el aporte de sombra, el aumento en biodiversidad, protección contra el viento, entre otros. Sin embargo, el enfoque sobre su aporte socioeconómico ha sido débilmente abordado [1], [11], [7], [17]. Por lo tanto, este estudio se ha enfocado en determinar los beneficios económicos directos que se pueden obtener del aporte de madera de cedro en un sistema agroforestal de café.

\section{Materiales y métodos}

El estudio se realizó en el cantón de Pérez Zeledón, provincia de San José, Pacífico central de Costa Rica (Figura 1). En esta región el cultivo del café es una de las actividades económicas principales con más de 12000 ha, en altitudes que van desde los 600 m.s.n.m. hasta poco más de los 1700 msnm [5].

Se eligieron aleatoriamente 26 fincas de productores asociados a Coopeagri R.L. con registros de establecimiento de plantaciones de cedro entre 10 a 16 años de edad. Estas fincas se encuentran sometidas al régimen de Pago de Servicios Ambientales (PSA) del Fondo de Financiamiento Forestal (FONAFIFO).

\section{Muestreo de fincas y mediciones de campo}

\section{Recolección de datos}

Coopeagri registra alrededor de 106 fincas de café, en 9 distritos del cantón, que cumplen con la condición de modalidad de SAF con cedro con más de 6 años de edad. La organización aglutina fincas en casi todo el Cantón de Pérez Zeledón, con mayor relevancia en los distritos San Isidro de El General, Platanares, Pejibaye, Cajón, Río Nuevo, La Amistad, San Pedro, Rivas y El General, que cumplen con la condición de modalidad de SAF con cedro con más de 10 años de edad. Se eligieron fincas en al menos tres de los 9 distritos mencionados (estratos o subpoblaciones), donde se escogieron aleatoriamente entre 10 y 15 fincas con sombra de cedro, que correspondió con un $25 \%$ de la muestra de fincas potenciales de cada uno de estos estratos.
En las fincas elegidas se tomó aleatoriamente un lote o rodal de café con cedro, con preferencia en aquellos donde se pudiera distinguir las líneas de plantación original de los árboles de cedro, con el propósito de facilitar el proceso de toma de datos. Se trabajó con el método de muestreo basado en árboles individuales, donde cada árbol es una parcela o unidad muestral [14]. En los lotes elegidos, se procedió a realizar un barrido completo, donde se censaron todos los árboles en pie.

A cada lote muestreado se le estimó el área efectiva plantada de café con cedro, por medio de un GPS Garner, utilizando la aplicación de "recorrido" a lo largo de la periferia de la superficie efectiva plantada. Con base en un número aleatorio inicial elegido, se localizó el primer árbol desde donde se continuó con el procedimiento de muestreo sistemático, tal y como lo definen Murillo y Badilla [14]. Se realizó el recorrido o barrido sistemático a través de todo el rodal, contabilizando todos los árboles de cedro presentes en pie. A cada décimo árbol se le midió y evaluó sus características de producción: DAP con corteza $(\mathrm{cm})$, altura comercial potencial estimada a partir del número de trozas de 2,5 m de largo, calificación de la calidad de cada una de las primeras cuatro trozas, según la metodología propuesta por Resende, Murillo y Badilla [18]. Esto implica que se utilizó una intensidad de muestreo del $10 \%$ de los árboles de cedro en pie en el lote. Los árboles de las orillas irregulares del lote o cualquiera no incluido en la contabilidad sistemática, fueron registrados como saldo y se agregaron en la

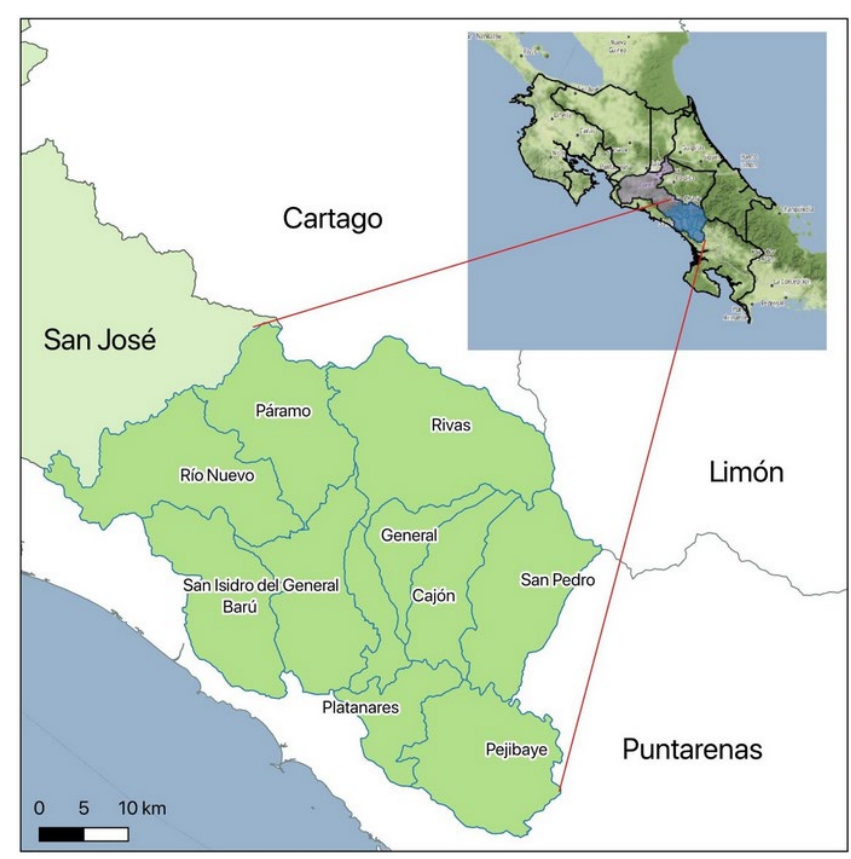

Figura 1. Localización del cantón de Pérez Zeledón en Costa Rica

Figure 1. Pérez Zeledón county location in Costa Rica 
cuenta final del total de árboles en pie del lote. De esta manera se obtuvo un censo de los árboles de cedro en pie en cada lote investigado.

Con ayuda del software para valuación de plantaciones forestales [19], se determinó el volumen comercial y el valor potencial de la madera en pie de cada individuo y colectivamente, en cada lote muestreado. El programa utiliza un algoritmo de conicidad para la estimación del volumen comercial, que se fundamenta en una serie de coeficientes de conicidad o factor de reducción diamétrico por metro lineal del fuste.

Para estimar el valor de la madera del árbol en pie, se clasificó cada una de sus posibles trozas en tres categorías de mercado, según su uso potencial con base en sus dimensiones y calidad de fuste: 1) leña, aquellas trozas de calidad 4 o con un diámetro $<$ a $10 \mathrm{~cm}$ en su sección menor; 2) tarima, aquellas trozas de calidad de 1 a 3, pero con un diámetro entre 10 y $20 \mathrm{~cm}$ en su sección menor; 3) madera para aserrío para las trozas de calidad de 1 a 3 , con un diámetro $>20 \mathrm{~cm}$ en su sección menor. Los precios de referencia utilizados se tomaron del valor del mercado de la madera de cedro en la región de Pérez Zeledón [20]. Se utilizó un precio de $\$ 5000 \mathrm{~m}^{3}$ estéreo (US $\$ 8,5)$ para la leña (piezas de 1,2 $\mathrm{m}$ de largo), con un factor de 0,58 para su conversión a madera sólida. El precio de la madera de tarima fue de $\$ 60 \mathrm{PMT}^{-1}$ (Pulgada Maderera Tica) y el de madera gruesa para aserrío en pie se estimó en $\$ 150$ PMT $^{-1}$. El valor del US dólar utilizado fue el predominante en el I semestre del 2019, que fue de \$575 por $1 \$$.

El espaciamiento promedio entre los árboles de cedro se obtuvo, al relacionar el área efectiva del cafetal estudiado, dividido por el número de árboles censados.

Con base en las mediciones de campo, se ajustó un modelo de crecimiento del DAP vs edad, con el programa Microsoft Excel [21]. En cada lote se obtuvo un promedio del DAP y de esta manera fue constituida la base de datos para el modelo de predicción del crecimiento DAP edad $^{-1}$ Con el fin de reducir la variabilidad entre fincas, producto de densidad de árboles diferente, se utilizó el valor promedio diamétrico de la finca como variable para el modelo DAP edad-1.

Con la base de datos completa y unificada se procedió con la construcción de un gráfico de DAP edad ${ }^{-1}$, con el fin de detectar datos sesgados y verificar que la variable dependiente (DAP), tuviese una buena relación con respecto a la variable independiente (edad). Los datos fueron analizados en Excel [21] mediante la opción de regresión, de donde se evaluaron de forma independiente todas las opciones de modelos. La selección del mejor modelo se realizó a través de los siguientes criterios estadísticos:
- Coeficiente de determinación $\left(\mathrm{r}^{2}\right)$ : Indica la proporción de la variación total observada en la variable dependiente, que es explicada por el modelo utilizado [22]. El coeficiente se calculó de la siguiente manera (ecuación 1).

$$
r^{2}=1-\frac{\sum(\text { Yobs }- \text { Yest })^{2}}{\sum(\text { Yobs }- \text { Ymed })^{2}}
$$

Donde:

yobs es cada uno de los valores observados de la variable dependiente

yest es cada uno de los valores de la variable dependiente estimados a través del modelo de regresión desarrollado

ymed es la media aritmética de la variable dependiente

- Error estándar de la medición (Syx), que se determinó según la ecuación 2.

$$
\text { Syx }=\sqrt{\left[\frac{\sum(Y o b s-Y e s t)^{2}}{n-p}\right]}
$$

$n$ es número de observaciones incluidas en el análisis.

$p$ es número de coeficientes en el modelo de regresión.

- Análisis de residuos: Se definió como un residuo (resd) a la diferencia entre el valor observado de la variable dependiente (Yobs) y el valor estimado o predicho por el modelo de regresión (Yest) [22] (Ecuación 3).

$$
\text { Resid }=\text { Yobs }- \text { Yest }
$$

Con las bases de datos de volumen y valor comercial por hectárea de cada uno de las 26 fincas muestreadas, se procedió también al ajuste de un modelo de predicción, edad/volumen comercial y otro para edad/ valor comercial. Los datos fueron analizados también en la hoja electrónica EXCEL [21] mediante la opción de regresión, tal y como se explicó para el primer modelo.

\section{Resultados}

En el cuadro 1 se describe la información silvicultural de cada una de las 26 fincas investigadas en los distritos de PérezZeledón. Se puede observar que los cafetales tienen 
Cuadro 1. Fincas de café con cedro muestreadas en los distritos de Platanares, Pejibaye, Cajón, San Isidro de El General, Río Nuevo, La amistad, San Pedro, Rivas y El General de Pérez Zeledón, Pacífico central de Costa Rica.

Table 1. Sampled coffee farms with cedar in Platanares, Pejibaye, Cajón, San Isidro del General, Río Nuevo, La amistad, San Pedro, Rivas and El General districts in Pérez Zeledón, Central Pacífic of Costa Rica.

\begin{tabular}{|c|c|c|c|c|c|c|c|}
\hline Lote & Localidad & Año & $\begin{array}{l}\text { Edad del } \\
\text { cedro }\end{array}$ & $\mathrm{N}$ & DAP promedio & $\begin{array}{l}\text { Espaciamiento } \\
(\mathrm{m})\end{array}$ & Altitud (msnm) \\
\hline 1 & Las Mesas & 2006 & 13 & 79 & 33,93 & 11,28 & 746 \\
\hline 2 & Las Mesas & 2006 & 13 & 122 & 32,70 & 9,05 & 750 \\
\hline 3 & Las Mesas & 2003 & 16 & 114 & 39,90 & 9,35 & 743 \\
\hline 4 & $\begin{array}{l}\text { Mollejones, } \\
\text { Platan }\end{array}$ & 2008 & 11 & 120 & 27,90 & 9,12 & 750 \\
\hline 5 & San Carlos & 2009 & 10 & 198 & 16,70 & 7,10 & 725 \\
\hline 6 & La Suiza & 2008 & 11 & 151 & 34,70 & 8,14 & 930 \\
\hline 7 & Mollejones & 2008 & 11 & 328 & 23,50 & 5,53 & 816 \\
\hline 8 & Villa Argentina & 2008 & 11 & 228 & 23,30 & 6,62 & 870 \\
\hline 9 & Bolivia & 2007 & 12 & 153 & 27,20 & 8,08 & 930 \\
\hline 10 & San Miguel & 2009 & 10 & 567 & 24,20 & 4,20 & 730 \\
\hline 11 & Cristo Rey & 2009 & 10 & 709 & 18,60 & 3,75 & 520 \\
\hline 12 & Cristo Rey & 2007 & 12 & 263 & 20,80 & 6,16 & 570 \\
\hline 13 & Cristo Rey & 2008 & 11 & 301 & 22,90 & 5,76 & 570 \\
\hline 14 & San Carlos & 2006 & 13 & 143 & 41,30 & 8,38 & 560 \\
\hline 15 & Las mesas & 2006 & 13 & 70 & 32,70 & 11,97 & 685 \\
\hline 16 & Santa Rosa & 2007 & 12 & 440 & 17,4 & 4,77 & 770 \\
\hline 17 & Santa Rosa & 2009 & 10 & 300 & 23,68 & 5,77 & 940 \\
\hline 18 & Santa Rosa & 2008 & 11 & 103 & 26,08 & 9,86 & 700 \\
\hline 19 & La Unión & 2008 & 11 & 338 & 21,67 & 5,44 & 600 \\
\hline 20 & Fátima & 2008 & 11 & 157 & 19,01 & 7,98 & 993 \\
\hline 21 & San Ignacio & 2009 & 10 & 130 & 26,34 & 8,76 & 900 \\
\hline 22 & Quizarrá & 2007 & 12 & 88 & 31,13 & 10,67 & 780 \\
\hline 23 & Quizarrá & 2007 & 12 & 107 & 33,54 & 9,66 & 780 \\
\hline 24 & Quizarrá & 2007 & 12 & 105 & 32,43 & 9,77 & 780 \\
\hline 25 & San Agustín & 2009 & 10 & 112 & 20,70 & 9,46 & 800 \\
\hline 26 & Tirrá & 2008 & 11 & 138 & 25,90 & 8,52 & 1090 \\
\hline
\end{tabular}

en promedio 214 árboles ha, en una amplia variación que va desde 70 hasta 700 árboles. Este valor promedio corresponde con un espaciamiento aproximado de $7,9 \mathrm{~m}$ o casi $8 \mathrm{~m}$ entre árboles. La totalidad de plantaciones investigadas se localizaron por debajo de los $950 \mathrm{msnm}$ (cuadro 1).

En la figura 2 se muestra el modelo ajustado para estimar el crecimiento del DAP $(\mathrm{cm})$ promedio con respecto a la edad (años), para árboles de cedro plantados a gran espaciamiento en cafetales (distanciamiento de $8 \times 8 \mathrm{~m}$ o más). Puede notarse el buen crecimiento de la especie, que logra alcanzar los $40 \mathrm{~cm}$ de diámetro a los 16 años. El ajuste del modelo mejoró notablemente al hacerlo pasar por el origen, a pesar de la enorme variación en crecimiento entre fincas con cedro de una misma edad.

En el cuadro 2 se muestra en detalle la información sobre el volumen y su valor comercial potencial, para cada una de las 26 fincas incluidas en la muestra. El valor total de la madera para aserrío (diámetro $>20 \mathrm{~cm}$ ) alcanzó casi los US \$6 500 ha-1 a los 12 años, mientras que el valor total alcanzó cerca de los \$ 7000 ha. El aporte del valor de la leña y la madera para tarimas (diámetros entre 10 y 20 $\mathrm{cm}$ ) no superó el $10 \%$ del valor total ha-1 Sin embargo, en términos de volumen, la leña registró en promedio un $37 \%$ del volumen total, la tarima un $6 \%$ y la madera para aserrío un $57 \%$. 


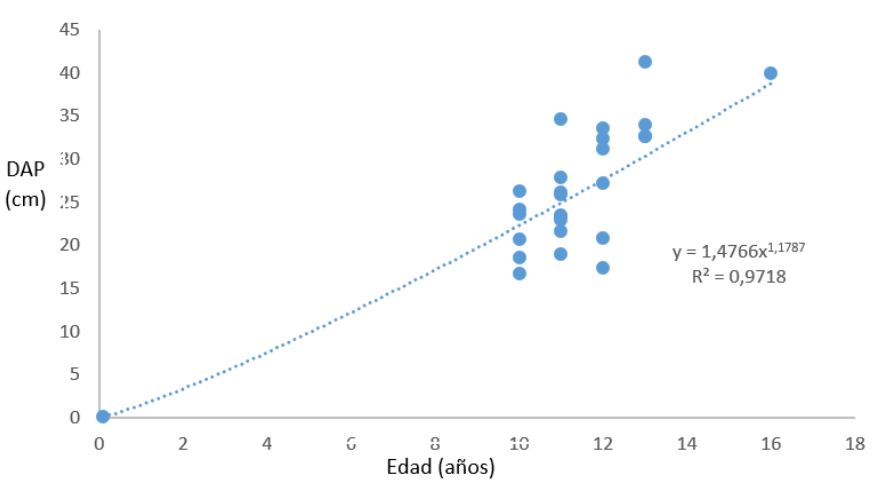

Figura 2. Relación del crecimiento del DAP-edad de cedro (Cedrela odorata) en sistemas agroforestales con café en Pérez Zeledón, Costa Rica.

Figure 2. DBH-age growth relationship of cedar (Cedrela odorata) in agroforestry systems with coffee in Pérez Zeledón, Costa Rica.

En la figura 3 se muestra la calidad de las trozas de 2,5 $\mathrm{m}$ del árbol tipo de cedro, presente en los cafetales de Pérez Zeledón. Se puede apreciar como se distribuye el potencial productivo de un árbol típico, que no ha recibido prácticamente ningún manejo silvicultural. La primera troza en general es de excelente calidad, con un valor de un $96 \%$ que la faculta totalmente para aserrío. La segunda troza tiene en promedio un $62 \%$ de calidad, que también permite su aprovechamiento en aserrío en la mayoría de los casos. Mientras que la tercera troza tiene en promedio, solamente un $21 \%$ de calidad para aserrío, lo que implica que solamente media troza es aprovechable para este uso. Estos valores implican que el árbol promedio de cedro tiene una altura comercial para aserrío, de aproximadamente 6 a $7 \mathrm{~m}$ de fuste $(2,5$ trozas comerciales de 2,5 m). El resto de su volumen tiene un mercado marginal como leña, aunque de magnitud volumétrica significativa. Cabe mencionar que, en árboles con más de 15 años tumbados en varias fincas, se observó empíricamente la presencia significativa de duramen, en más de un $60 \%$ del volumen de la primera troza.

El modelo de crecimiento y los datos de campo claramente evidencian, que es posible aumentar la productividad e impacto económico si se optimiza la densidad de árboles en el cafetal. Las distancias de siembra del café usualmente son de $2 \times 1 \mathrm{~m}$, sin embargo, el ICAFE viene promoviendo la mecanización de los cafetales a través de un distanciamiento de 2,5 $\mathrm{m} \times 1,8 \mathrm{~m}$, para mantener un $\mathrm{N}=5000$ plantas de café ha-1 [23], [4]. Esto implica que un escenario realista podría fomentar un aumento en la siembra de árboles de cedro, con 267 árboles ha ${ }^{-1}$ al inicio $(5 \times 7,5 \mathrm{~m})$, para luego ralear un $40 \%$ a los 8 años

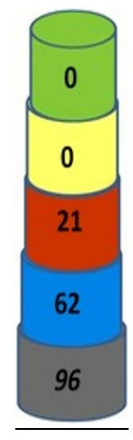

Figura 3. Calidad y potencial comercial (\%) para aserrío de trozas de 2,5m de longitud, del árbol tipo de cedro amargo, de 10 a 16 años de edad, en cafetales de Pérez Zeledón.

Figure 3. Quality and commercial potential (\%) for sawmill in $2.5 \mathrm{~m}$ length-logs, in a typical cedar tree of 10 to 16 years-old, in coffee farms in Pérez Zeledón.

y dejar $\mathrm{N}=160$ árboles en pie que continuarían hasta su cosecha final a los 16 años. En cafetales ya establecidos, los cedros se pueden plantar a $6 \times 6 \mathrm{~m}(\mathrm{~N}=278)$, para luego ralear un $50 \%$ y dejar en pie $\mathrm{N}=139(12 \times 6 \mathrm{~m})$. Los árboles deberán ser descumbrados (poda de copa) cada uno o dos años para mantener su tamaño de copa y evitar la sombra excesiva en el período lluvioso [24], [25]. En el cuadro 3 se muestra el resultado que se podría obtener con este sistema de plantación de cedro en cafetales, con su respectivo crecimiento y rendimiento esperado. El ingreso esperado por la madera para el caficultor sería de US $\$ 6140+\$ 260$ (40\% del raleo al año 8) = \$6400 ha-1 y un volumen comercial de $65 \mathrm{~m}^{3}+$ $11,2 \mathrm{~m}^{3}=76,25 \mathrm{~m}^{3} \mathrm{ha}^{-1}$.

En la figura 4 se observa el mejor modelo ajustado que permite predecir el volumen comercial ha- 1 vs la edad de los árboles de cedro en cafetales. Se puede observar que el modelo exponencial tuvo el mejor ajuste, a pesar de la gran variación en el número de árboles de cedro ( $\mathrm{N}$ desde 70 hasta 700 ) entre fincas, que explica la dispersión de los datos de volumen de cedro entre fincas, a una misma edad de los árboles. El modelo ajustado determina, que en promedio a los 16 años los árboles de cedro producirán poco más de $105 \mathrm{~m}^{3} \mathrm{ha}^{-1}$.

En la figura 5 se muestra un modelo que predice el valor esperado de la madera de cedro en un cafetal, vs la edad de los árboles. Puede observarse que el modelo exponencial fue nuevamente el de mejor ajuste, que al hacerlo pasar por el origen, registró una mejor explicación de la variación observada. Nuevamente, la gran variación en el número de árboles de cedro por finca ( $\mathrm{N}$ desde 70 hasta 700 ), explica la dispersión del 
Cuadro 3. Crecimiento estimado y valor económico en pie de madera de cedro (Cedrela odorata), con una densidad inicial de $\mathrm{N}=267$ ( $5 \times 7,5 \mathrm{~m}$ ) y un raleo de 107 árboles (año 8), en sistemas agroforestales de café, Pérez Zeledón, Costa Rica. Número de árboles ha-1 (N) se anota en paréntesis en la columna de edad.

Table 3. Estimated growth and standing wood economical value of cedar (Cedrela odorata), under an initial density of $\mathrm{N}=267(5 \times 7.5 \mathrm{~m})$ and a thinning of 107 trees (age 8), in agroforestry systems with coffee, Pérez Zeledón, Costa Rica. Number of trees ha-1 $(\mathrm{N})$ has been noted in parenthesis at the age column.

\begin{tabular}{|c|c|c|c|c|c|c|c|c|c|}
\hline Edad (N) & $\mathrm{DAP}(\mathrm{cm})$ & $\begin{array}{l}\text { Vol. Com. } \\
\text { Aserrío } \\
\left(\mathrm{m}^{3} h \mathrm{~h}^{-1}\right)\end{array}$ & $\begin{array}{l}\text { Valor } \\
\text { Aserrío } \\
\left(\$ \text { \$h }^{-1}\right)\end{array}$ & $\begin{array}{c}\text { Vol. Com. } \\
\text { Tarima } \\
\left(m^{3} h a^{-1}\right)\end{array}$ & $\begin{array}{l}\text { Valor } \\
\text { Tarima } \\
\left(\$ h a^{-1}\right)\end{array}$ & $\begin{array}{c}\text { Vol. Com. } \\
\text { Leña } \\
\left(m^{3} h a^{-1}\right)\end{array}$ & $\begin{array}{l}\text { Valor Leña } \\
\left.\left(\$ \text { \$ha }^{-1}\right)\right)\end{array}$ & $\begin{array}{c}\text { Vol. Com. } \\
\text { Total } \\
\left(\mathrm{m}^{3} h a^{-1}\right)\end{array}$ & $\begin{array}{c}\text { Valor Total } \\
\left(\text { (\$ha }^{-1}\right)\end{array}$ \\
\hline $4(267)$ & 11,46 & 0 & 0 & 0 & 0 & 9,02 & 152 & 9,02 & 152 \\
\hline $8(267)$ & 15,54 & 0 & 0 & 5,51 & 208 & 13,24 & 225 & 18,74 & 433 \\
\hline 12 (160) & 29,67 & 23,90 & 1940 & 0,0 & 0,0 & 18,32 & 345 & 43,20 & 2285 \\
\hline 16 (160) & 40,49 & 65,00 & 6140 & 0,0 & 0,0 & 57,70 & 1090 & 122,70 & 7230 \\
\hline
\end{tabular}

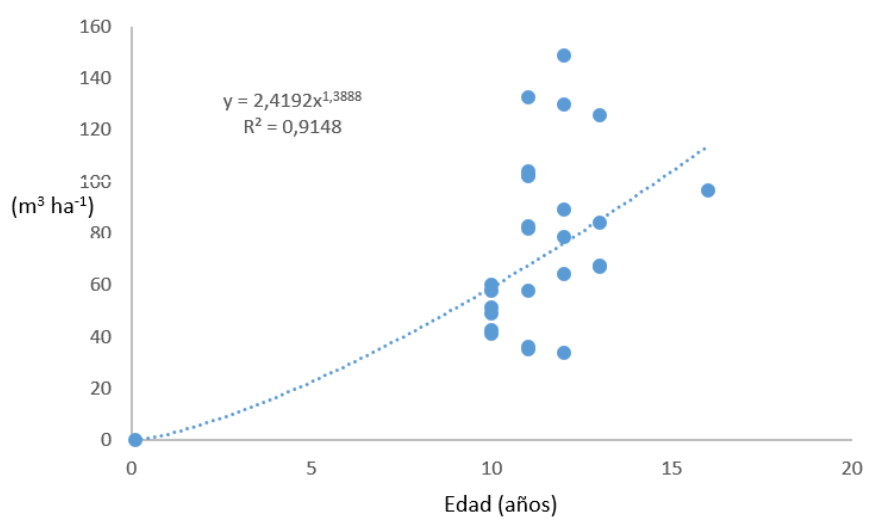

Figura 4. Relación entre la edad y el volumen comercial total en pie $\left(\mathrm{m}^{3} \mathrm{ha}^{-1}\right)$ de madera de cedro en cafetales de Pérez Zeledón ( $\mathrm{N}$ promedio de $214 \pm 10,6)$.

Figure 4. Age and total standing commercial volume relationship $\left(\mathrm{m}^{3} \mathrm{ha}^{-1}\right)$ of cedar wood in Pérez Zeledón coffee farms (average $\mathrm{N}$ of $214 \pm 10.6)$.

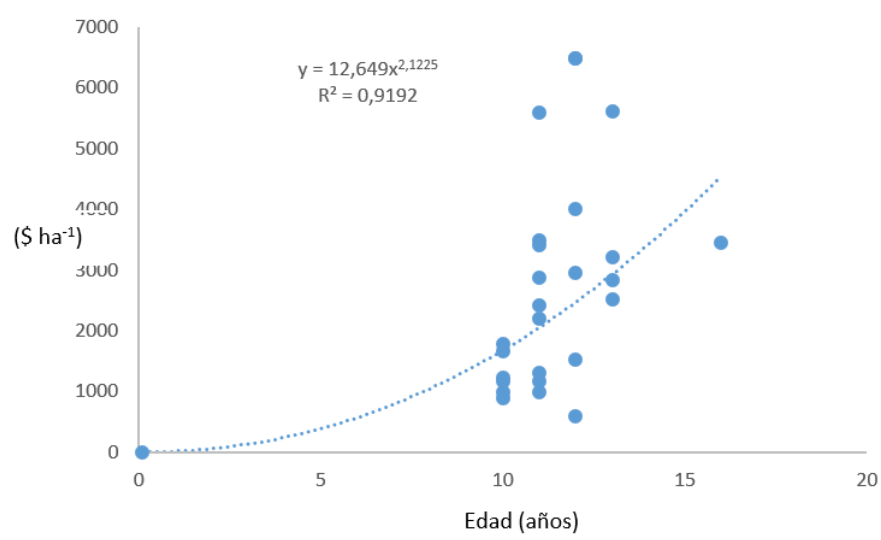

Figura 5. Relación entre la edad y el valor de la madera total en pie (US\$) en árboles de cedro en cafetales de Pérez Zeledón (N promedio de $214 \pm 10,6)$

Figure 5. Age and standing total wood value (US\$) in cedar trees in Pérez Zeledón coffee farms (average $\mathrm{N}$ of $214 \pm 10.6$ ). valor de la madera en pie entre fincas con árboles de una misma edad. El modelo en promedio determina, que a los 16 años el valor de la madera de cedro alcanzará casi los $\$ 5000$ ha $^{-1}$.

\section{Discusión}

De manera general, se registró una fuerte variación en la densidad de árboles en pie entre fincas, que iban desde los 70 hasta los 700 individuos ha-1. Estos resultados afectaron sensiblemente el ajuste de los modelos de predicción del crecimiento en DAP, del volumen comercial y del valor económico por hectárea, como se podría esperar. Sin embargo, los datos si permitieron obtener un patrón definido del comportamiento y rendimiento comercial del árbol de cedro en el cafetal en esta región del país. Para Costa Rica algunos autores han reportado densidades de cedro de $\mathrm{N}=50$ a 128 árboles ha $^{-1}$ [24], de 40 a 500 [3], 68 a 605 [5]. Valores similares a los registrados en esta investigación, que oscilaron de 70 a 700 . Sin embargo, por lo general estos estudios se han basado en parcelas de muestreo de tamaño fijo como $500 \mathrm{~m}^{2}$, lo que puede conducir a errores de estimación, dada la irregularidad en la distancia entre los árboles en cada cafetal. Lo que podría haber generado una variación significativa en el número de árboles entre parcelas de muestreo. Mientras que el sistema de muestreo basado en árboles individuales empleado en este estudio, permitió obtener un censo del total de individuos en pie. El levantamiento exacto del área efectiva de cada lote investigado, permitió determinar con precisión la relación, cantidad de árboles con el área exacta del cafetal. Una muestra de 26 fincas en distintas regiones de Pérez Zeledón, permite establecer, una base estadística suficiente y representativa de los cafetales de este cantón. 
Salgado [7] recomienda finalizar con 70 a 156 individuos por hectárea en plantaciones de maderables en café. Sin embargo, esta relación debe estar en función del diámetro de copa de los árboles y el efecto de su sombra en el periodo lluvioso en los cafetos, tema del que no se reporta suficiente información a este nivel de detalle [25]. La sombra de la copa irá aumentando con la edad, tema que debe formar parte del manejo de los árboles. Prácticas como la descumbra o descope a una altura comercial deseada, junto con el control de la Hypsipylla grandella los primeros dos a tres años, son dos actividades fundamentales que deben ser incorporadas al paquete tecnológico por desarrollar en este SAF. Este tipo de manejo silvicultural de los árboles, podría permitir mantener una mayor densidad de árboles ha-1, tal vez un número inicial de $\mathrm{N}=267$, con un mucho mayor impacto económico para los productores, tal y como se propone en el cuadro 3.

La edad también ejerció un efecto importante en los resultados. En plantaciones con más de 10 a 12 años, se observó que con frecuencia tuvieron raleos o alguna cosecha parcial de parte de la población inicial de árboles, donde con frecuencia, a menudo se habrían extraído los mejores individuos del lote. Esto pudo provocar una fuerte variación en la cantidad de árboles en pie, que repercutió en la estimación del volumen comercial por hectárea. Sin embargo, a pesar de esta gran variación, los datos permitieron ajustar modelos aceptables que permiten mostrar el comportamiento del crecimiento promedio del cedro en cafetales, con un $\mathrm{N}$ promedio de $\mathrm{N}=214 \pm 10,6$.

El modelo de crecimiento muestra que el cedro en esta región, alcanza los $40 \mathrm{~cm}$ a los 16 años de edad. Estos resultados concuerdan casi exactamente con lo estimado por la investigación de González et al. [3] en esta misma región, así como el crecimiento general esperado para el cedro plantado en el país (DAP $=37 \mathrm{~cm}$ ), propuesto por Murillo y Badilla [14]. Dado el espaciamiento amplio entre árboles, podría asegurarse que la tasa de crecimiento diamétrico estuvo sin competencia durante la mayor parte de los años. Mientras que Jiménez [1] reporta $32 \mathrm{~cm}$ a los 16 años de edad en árboles de cedro en cafetales de Honduras. En plantaciones de cedro en Guatemala se reporta una tasa anual de crecimiento del DAP de entre 2 y $3 \mathrm{~cm}$ año-1 para sitios en sistemas agroforestales [12]. Por tanto, estos resultados pueden considerarse como fidedignos, representativos y muestran el enorme potencial productivo del cultivo de madera de cedro en cafetales.

Con relación al crecimiento del volumen comercial, se observó un valor máximo de $148 \mathrm{~m} 3$ a la edad de 12 años, con una densidad de $\mathrm{N}=88(10,6$ × 10,6m). La variación en volumen entre sitios de una edad parecida, se explica por la realización de raleos o cosechas parciales por lo alto, practicada por las necesidades de los cafetaleros. Sin embargo, en la figura 5 se muestra un buen modelo de predicción del volumen comercial con la edad de los árboles. Este modelo estima que a los 16 años se obtendrá un volumen comercial total de poco más de $110 \mathrm{~m}^{3} \mathrm{ha}^{-1}$, que es superior a los $92 \mathrm{~m}^{3}$ reportados por González et al. [3] y a los $100 \mathrm{~m}^{3}$ reportados por Jiménez con cedro en cafetales en Honduras a los 30 años de edad [1]. La variación en estos volúmenes se explica principalmente por las diferencias en el número de árboles ha- ${ }^{-1}(\mathrm{~N})$ entre los distintos estudios, producto de sistemas de muestreo también diferentes.

En el cuadro 3 se muestra una opción de producción con una mayor densidad inicial de árboles de cedro. En este ejemplo, se lograría una producción de más de $76 \mathrm{~m}^{3} \mathrm{ha}^{-1}$ de volumen comercial aserrable, que sumado al volumen de leña, alcanzaría los $122 \mathrm{~m}^{3} \mathrm{ha}^{-1}$. Para la estimación del volumen comercial, en este estudio se utilizó un software de valuación, que permite realizar un despiece del árbol y valorar no solo la madera comercial para aserrío, sino también el volumen marginal que pueda ser utilizado en el mercado de la tarima y el de la leña. Debe mencionarse que este volumen comercial aserrable se estimó para un diámetro mínimo de $18 \mathrm{~cm}$ en la cara menor y con base en el método de mecate o pulgada maderera tica. Que es un estimado de volumen realista, tal y como se utiliza en el mercado local. Con lo cual, se logró un estimado de mayor precisión del volumen comercial por árbol.

El volumen de la leña aportaría un $45 \%$ del volumen total, sin embargo, solamente participaría en un $15 \%$ del valor comercial del árbol. Esto volumen adicional, aunque marginal en valor, podría explicar esa diferencia en volumen comercial obtenido en este estudio, en comparación con los reportado por los otros investigadores mencionados. El volumen en leña es significativamente alto en árboles de cedro, debido a que no recibieron ningún manejo del control del barrenador del meristemo, ni podas. Lo que produce un árbol con muy poca altura comercial para aserrío (solamente dos trozas completas y una tercera troza corta), tal y como se observa en la figura 3.

La variación registrada de los datos entre fincas fue sumamente alto, lo cual puede explicar que el modelo exponencial se ajustara mejor que un modelo sigmoidal, como usualmente se espera conforme envejece la plantación. El obligar a pasar la función por el origen, tiene sentido biológico, pero pudo influir en que el mejor modelo ajustado, mostrara aún una pendiente creciente sostenida a los 16 años de edad. 
El modelo de predicción del valor de la madera de cedro vs edad, registró un muy buen ajuste, similar al del modelo del volumen comercial. Ambos modelos están estrechamente ligados, tal y como se espera. Su gran variación obedece a la misma dinámica explicada, donde los dueños del cafetal toman el recurso maderable cada vez que los apremie alguna situación socio-económica en su economía de finca. Este es el principio esperado de los beneficios de un buen SAF. Tal y como se explicó con el modelo de volumen, la amplia variación de resultados entre fincas y el paso del modelo por el origen, explica la razón del ajuste de un modelo exponencial, con una pendiente creciente a pesar de los 16 años de edad. Los resultados registran una tasa de incremento de $\$ 400$ ha $^{-1}$ año-1 (IMA) sin manejo. Con la incorporación de técnicas de manejo de la copa, poda de formación, de fumigación los primeros dos años para aumentar la altura comercial a unos 7,5 m (3 trozas comerciales por árbol), se podría aumentar la densidad de árboles y con esto, se podría aumentar significativamente los ingresos a los productores. Así también, con el uso de material genético de rápido crecimiento, selección temprana por su mayor dominancia apical [26], junto con un buen manejo del ataque de Hypsipylla grandella, podría aumentarse la densidad del cultivo de cedro en el cafetal y la producción de madera comercial en general.

\section{Conclusiones}

El cultivo de cedro en los cafetales de Pérez Zeledón produce ingresos económicos por la madera, de aproximadamente $\$ 5000 \mathrm{ha}^{-1}$ y un volumen comercial

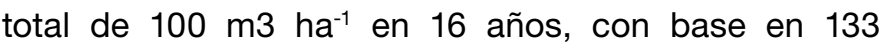
individuos ha-1. El valor de la madera de cedro podría superar los $\$ 7000 \mathrm{ha}^{-1}$ si se realiza un mayor control del barrenador del meristemo y con una densidad inicial de 267 árboles ha-1.

El cedro en cafetales tiene una excelente tasa de crecimiento del DAP de $2,5 \mathrm{~cm}^{*} a n ̃ o^{-1}$, que le permite alcanzar los $40 \mathrm{~cm}$ en el DAP a los 16 años de edad.

El cultivo de cedro en cafetales de Pérez Zeledón se maneja a una densidad empírica promedio de 214 individuos ha ${ }^{-1}$, que corresponde con un espaciamiento de $7 \mathrm{~m} \times 7 \mathrm{~m}$. Esta densidad de árboles podría revisarse y hasta aumentarse, con el propósito de aumentar los indicadores de productividad de los cafetales.

El árbol tipo cultivado de cedro tiene una altura comercial para aserrío, de aproximadamente 6 a $7 \mathrm{~m}$ de fuste, que corresponde con dos trozas comerciales de $2,5 \mathrm{~m}$ cada una y, una a dos trozas cortas de 1,2m para tarima o leña.

\section{Agradecimientos}

Este estudio tuvo financiamiento de la Vicerrectoría de Investigación y Extensión del Instituto Tecnológico de Costa Rica, como parte del proyecto de extensión "Diseño de sistemas de producción de café asociado con maderables nativos de alto valor".

Se agradece el apoyo de Coopeagri en la logística, información de las plantaciones y durante el trabajo de campo.

\section{References}

[1] N.G. Jiménez. "Producción de madera y almacenamiento de carbono en cafetales con cedro (Cedrela odorata) y caoba (Swietenia macrophylla) en Honduras". Tesis M.Sc. CATIE, Turrialba, Costa Rica. 2012.

[2] Instituto Nacional de Estadística y Censo (INEC). VI Censo Nacional Agropecuario. San José, Costa Rica. 2015. Disponible en: http://www.mag.go.cr/bibliotecavirtual/ a00338.pdf.

[3] M. González-Rojas, R. Murillo-Cruz, E. Virginio-Filho, y C. Ávila-Arias. "Influencia de factores biofísicos y de manejo en el crecimiento de Cedrela odorata L. en asocio con café en Pérez Zeledón, Costa Rica,”. Revista Forestal Mesoamericana Kurú, 15(36), 46-58. 2017.

[4] H. Castillo, P. Montoya, Y. Badilla, O. Murillo. "Diseños para incorporar maderables de alto valor en SAF café". Presentado en I Simposio Cultivo Especies Forestales Nativas. Estación Forestal Experimental Horizontes, Liberia, Guanacaste, 5-6 diciembre. 2017.

[5] M. González-Rojas, R. Murillo-Cruz, C. Ávila. "Rentabilidad financiera de Cedrela odorata L. en sistemas agroforestales con café en Pérez Zeledón," Costa Rica. Revista de Ciencias Ambientales Vol 52(1): 129-144. 2018.

[6] B. Dzib. "Manejo, secuestro de carbono e ingresos de tres especies forestales de sombra en cafetales de tres regiones contrastantes de Costa Rica". Tesis M.Sc. CATIE, Turrialba, Costa Rica. 2003.

[7] J.L. Salgado. "Fijación de carbono en biomasa aérea y rentabilidad financiera de sistema agroforestales con café en Turrialba, Costa Rica y Masatepe, Nicaragua". Tesis M.Sc. CATIE, Turrialba, Costa Rica. 2010.

[8] A. Jiménez. El café en Costa Rica. Gran modelador del costarricense. Editorial Universidad de Costa Ria. San José, Costa Rica. 692 p. 2013.

[9] F. Farfán. "Árboles con potencial para ser incorporados en sistemas agroforestales con café". FNC-CENICAFÉ. Bogotá, Colombia. 87 p. 2012.

[10] S. Sánchez, M.A. Mendoza, R.V. García. "Diversificación de la sombra tradicional de cafetales en Veracruz mediante especies maderables", Revista Mexicana de Ciencias Forestales Vol 8 (40): 7-17. 2017. 
[11] E. López-Sánchez, M.A. Musálem. "Sistemas agroforestales con cedro rojo, cedro nogal y primavera, una alternativa para el desarrollo de plantaciones forestales comerciales en los Tuxtlas, Veracruz, México". Revista Chapingo Serie Ciencias Forestales y del Ambiente 13(1): 59-66. 2007.

[12] Instituto Nacional de Bosques. "Paquete Tecnológico Forestal para Cedro Cedrela odorata L. Guatemala". Departamento de Investigación Forestal. 87p. (Serie técnica DT-029-2019). 2019.

[13] ONF (Oficina Nacional Forestal). Usos y aportes de la madera en Costa Rica. Estadísticas 2018 \& Precios 2019. San José, Costa Rica. 43 p. 2018.

[14] O. Murillo, Y. Badilla. Consultoría. "Definición de una metodología de muestreo de contratos del programa de pago de servicios ambientales para la medición de la biomasa, para el desarrollo de proyectos de comercialización de créditos de carbono". FONAFIFO. San José, Costa Rica. 108 p. 2015.

[15] A.L. Chacón. "Con soluciones orgánicas combaten la roya del café". Colegio de Ingenieros Agrónomos de Costa Rica. Revista Germinar 6(18):14-15. 2016.

[16] A. Nieters, J. Grabas, G. Jiménez, W. Alpízar. "NAMA café de Costa Rica - Una herramienta para el desarrollo bajo en emisiones". NAMA Facility Technical Support Unit. GIZ/ MINAE/MAG/ICAFE/DCC/Fundecooperación. San José, Costa Rica. 2015.

[17] M. González-Rojas. "Crecimiento y aporte financiero del cedro (Cedrela odorata L.) en sistemas agroforestales con café en Pérez Zeledón, Costa Rica". Tesis Lic. Universidad Nacional, Heredia, Costa Rica. 57 p. 2017.

[18] M.D.V. Resende, O. Murillo, Y. Badilla. "Selección de árboles plus" in Genética Cuantitativa y Selección en el Mejoramiento Forestal. Editorial Tecnológica de Costa Rica. Cartago, Costa Rica. 2018. Capítulo III, 25-45.

[19] O. Murillo, Y. Badilla. "Calidad y valoración de plantaciones forestales". Manual y Software. Instituto Tecnológico de Costa Rica. Escuela de Ingeniería Forestal. Cartago, Costa Rica. 51 p. 2004.

[20] D. Córdoba. "Precios de la madera de cedro en Pérez Zeledón en el 2019”. Personal e-mail (Junio 10, 2019).

[21] EXCEL Microsoft Office. Versión 2019. USA.

[22] E. Ortíz. "Crecimiento y rendimiento forestal". Manual. Instituto Tecnológico de Costa Rica, Escuela de Ingeniería Forestal. 2011.

[23] O. Murillo. "Diseños para aumentar el aporte económico del componente forestal en sistemas silvopastoriles y en café arbolado". Presentado en I Congreso Internacional de Vainilla y Sistemas Agroforestales. Santa Clara, San Carlos. Costa Rica. 7-11 marzo. 2016.

[24] J. Beer, R. Muschler, R., Kass, D., E. Somarriba. "Shade management in coffee and cacao plantations", Agroforestry systems, 38(1-3), 139-164. 1997.

[25] J. Hernández. "Incidencia de enfermedades foliares del café bajo diversos tipos de sombra y manejo de insumos, en sistemas agroforestales". Tesis Lic. Instituto Tecnológico de Costa Rica: Cartago Costa Rica, 75 pp. 2010.
[26] J.P. Cornelius, A.D. Watt. "Genetic variation in a Hypsipylaattacked clonal trial of Cedrela odorata under two pruning regimes", Forest Ecology and Management 183: 341-349. 2003. 